\title{
Single step production of Cas 9 mRNA for zygote injection
}

Bethany K Redel ${ }^{1}$, Benjamin P Beaton ${ }^{1,2}$, Lee D Spate ${ }^{1}$, Joshua A Benne ${ }^{1}$, Stephanie L Murphy ${ }^{1}$, Chad W O'Gorman', Anna M Spate ${ }^{1}$, Randall S Prather ${ }^{1}$ and Kevin D Wells ${ }^{1 *}$

${ }^{1}$ Division of Animal Science, National Swine Resource and Research

Center, University of Missouri, Columbia, MO, USA

${ }^{2}$ Current address, Genus plc, DeForest, WI, USA

BioTechniques 64:118-124 (March 2018) doi 10.4155/btn-2017-0116

Keywords:genetic modification; Cas9; zygote

Production of Cas 9 mRNA in vitro typically requires the addition of a $5^{\prime}$ cap and $3^{\prime}$ polyadenylation. A plasmid was constructed that harbored the T7 promoter followed by the EMCV IRES and a Cas9 coding region. We hypothesized that the use of the metastasis associated lung adenocarcinoma transcript 1 (Malat1) triplex structure downstream of an IRES/Cas9 expression cassette would make polyadenylation of in vitro produced mRNA unnecessary. A sequence from the mMalat1 gene was cloned downstream of the IRES/Cas9 cassette described above. An mRNA concentration curve was constructed with either commercially available Cas9 mRNA or the IRES/ Cas9/triplex, by injection into porcine zygotes. Blastocysts were genotyped to determine if differences existed in the percent of embryos modified. The concentration curve identified differences due to concentration and RNA type injected. Single step production of Cas9 mRNA provides an alternative source of Cas 9 for use in zygote injections.

With the rise of the CRISPR/Cas 9 technology, we have constructed a new Cas9 mRNA that does not possess a poly $(A)$ tail but rather a triple helical tail originating from the mMalat1 gene. The use of the triple helix tail lends itself to produce an unlimited, inexpensive Cas9 mRNA that creates modifications at the same efficiency as commercially available Cas9 mRNA. In the absence of polyadenylation, the resulting mRNA has a defined, fixed length that facilitates assessment of mRNA degradation.
Since the development and use of the CRISPR/Cas9 system, genetic engineering in zygotes of mammals is becoming highly efficient [1-5]. However, in vitro production of Cas9 mRNA has its limitations. After in vitro transcription, it is difficult to assess RNA degradation due the variable length of the poly $(A)$ tail. An alternative source of stable, assessable Cas9 mRNA may be more useful and more efficient for laboratories that produce their own Cas9 as compared to commercially produced
Cas9 mRNA that can be expensive and difficult to evaluate.

The majority of mRNAs are polyadenylated to possess a poly $(A)$ tail. This poly $(A)$ tail generally contains around 200 adenosine (A) nucleotides and is critical for the transcript to be exported out of the nucleus, translated, and is important for RNA stability. Similar to Malat1, histone mRNAs end in a six-base stem and four base loop that functionally replaces the poly(A) tail [6]. Surprisingly, recent research found that the 3 ' end of a nuclear noncoding RNA, Malat1, can also support the translation of a GFP reporter gene just as efficiently as the poly(A) tail [7]. While Malat1 is a long non-coding RNA (IncRNA), it is among the most abundantly expressed IncRNAs and the $A$ rich triple helical structure is able to bind to the ribosome and protect it from exonucleases. Addition of a triple helical structure to the Cas9 instead of the $\operatorname{poly}(A)$ tail simplifies mRNA production.

To create the triple helical structure on Cas9, a triple helical sequence from the mMalat1 gene was cloned into the plasmid vector pCR ${ }^{\mathrm{TM}} 4$-TOPO 9 (Invitrogen, Thermo Fischer, Grand Island, NY, USA) (pBKR1). pBKR1 was cut with Ncol to BstEll, gel purified, and cloned into a T7 IRES expression plasmid (pSF-T7-EMCV, Oxford Genetics, Oxford, UK) at Ncol to BstEll (pBKR2). Cas9 was cut $\mathrm{Ncol}$ to EcoRl from pX330 [8], gel purified, and cloned into pBKR2 at $\mathrm{Ncol}$ to EcoRl to make pBKR3 (Figure 1A). The pBKR3 plasmid was linearized with $B s m B I$ and in vitro transcription was completed by using the mMessage mMachine kit (Thermo Fisher). The kit utilizes an anti-reverse cap analog during transcription. The RNA was purified with a MEGAclear kit (Thermo Fisher). The Cas9 RNA that contains a triple helical tail is referred to as ' $M U$ Cas9 mRNA' (Figure1B). Alternatively, an uncapped mRNA was also produced by using the linearized $\mathrm{pBKR} 3$ vector and completing in vitro transcription by using a ribonucleotide solution mix (NEB, Ipswich, MA,

\section{METHOD SUMMARY}

A new Cas9 mRNA that contains an EMCV IRES and triple helical tail structure can be used for zygote injections. This mRNA can be produced in a single step without the addition of a poly(A) tail and maintains the same efficiency of modification as commercially available Cas9 mRNA. 
(A)

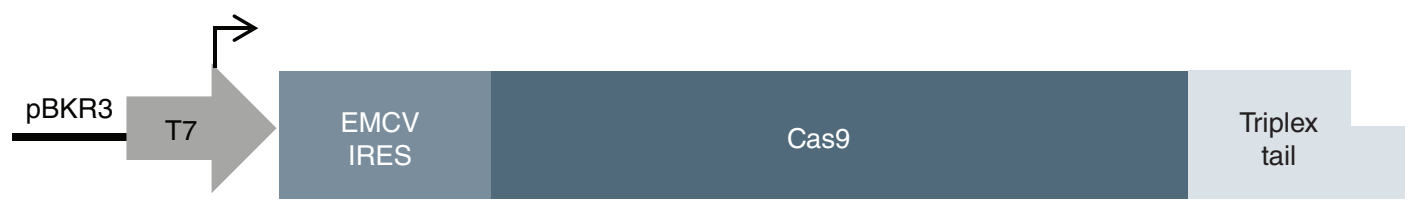

(B)

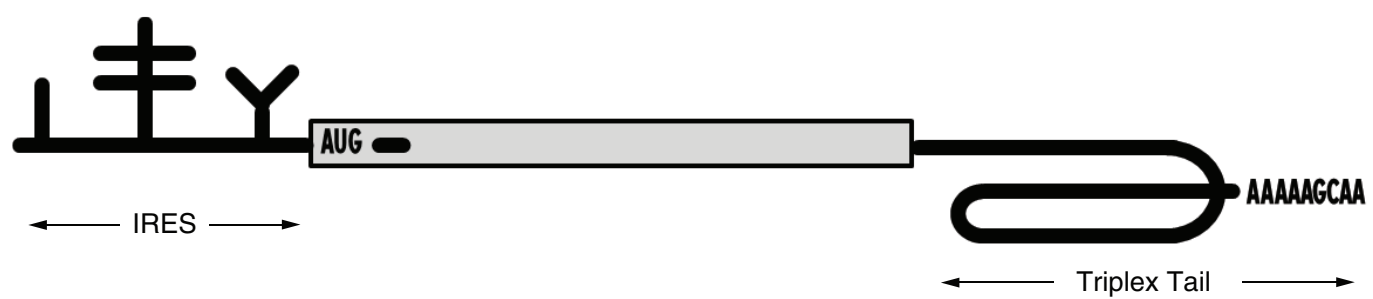

(C)

Figure 1. Diagrams of the components of the new method. A. Schematic diagram of a linearized pBKR3 vector. B. MU Cas9 mRNA_NC with specific elements identified. C. Cas9 containing a 5' cap and modified with pseudouridine and 5-methylcytidine mRNA containing a 3' poly(A) tail (TriLink Biotechnologies).

USA) and an RNA polymerase (NEB), and then the resulting mRNA was purified by using the MEGAclear kit. This uncapped mRNA was named MU Cas9 mRNA_NC. All guide RNAs (gRNAs) were produced as in Whitworth et al. [1]. Briefly, the $20 \mathrm{bp}$ guides were designed to target sequences next to an S. pyogenes protospacer adjacent motif [9]. A gBlock containing a T7 promotor sequence was added upstream of the guide, which was synthesized by Integrated DNA

Technologies (Coralville, IA, USA). Each gBlock was PCR amplified and then purified by using a Qiagen (Valencia, CA, USA) PCR purification kit. The purified gBlock amplicons were used for in vitro transcription by using the MEGAshortscript (Ambion, Thermo Fisher) kit. The resulting RNAs were then purified by using the MEGAclear Transcription Clean-Up Kit. Single guide RNA and Cas9 mRNA were diluted in nuclease-

Table 1. Percentage of modified blastocysts developed from zygote injections of commercially available Cas9 or MU Cas9 at varying concentrations. Five replicates of injections were completed to generate this data.

\begin{tabular}{|l|l|l|}
\hline \multicolumn{2}{|c|}{ Cas9 Source } & $\begin{array}{c}\text { Percentage of Modified } \\
\text { Blastocysts } \pm \text { S.E. }\end{array}$ \\
\hline $10 \mathrm{ng} / \mu \mathrm{L}$ MU Cas9 & $72.7 \% \pm 10.8 \%$ & 52 \\
\hline $10 \mathrm{ng} / \mu \mathrm{L}$ Cas9 & $73.0 \% \pm 7.5 \%$ & 23 \\
\hline $20 \mathrm{ng} / \mu \mathrm{L}$ MU Castocysts Assayed
\end{tabular}

Table 2. $P A H$ guide oligos and primer sequences used for genotyping single embryos.

\begin{tabular}{|l|l|}
\hline \multicolumn{2}{|c|}{ Oligo or Primer } \\
\hline gRNA1 & \multicolumn{1}{c|}{ Sequence } \\
\hline gRNA2 & GTCTATGGCAGAACAAAACTA \\
\hline Forward & CTTGTCTTGCTTCCAAGAAA \\
\hline Reverse & CACAGAACACGCCACTTAT \\
\hline
\end{tabular}

free water and stored at $-80^{\circ} \mathrm{C}$ until zygote injection.

An mRNA concentration curve was completed to determine the optimal concentration of Cas9 mRNA to be used during zygote injection with respect to both blastocyst development and the percent gene edited. Four proprietary gRNAs that were previously confirmed to create modifications during zygote injections were used to test for the efficiency of modifications. MU Cas9 mRNA was directly compared with commercially available Cas 9 mRNA that had been polyadenylated and contains 5-methylcytidine and pseudouridine modifications (TriLink Biotechnologies, San Diego, CA, USA) (Figure 1C). Cas9 mRNA (10 ng/ $\mu \mathrm{l}$, $20 \mathrm{ng} / \mu \mathrm{l}, 40 \mathrm{ng} / \mu \mathrm{l}$, and $80 \mathrm{ng} / \mu \mathrm{l})$ were each co-injected with all four gRNAs (15 $\mathrm{ng} / \mathrm{ul}$ ). Zygotes were created by using in vitro matured oocytes and in vitro fertilization [1]. The injected zygotes were cultured in MU2 medium for 7 days [10].

Development to the blastocyst stage was reduced by injecting zygotes with either Cas 9 mRNA compared to zygotes that were not injected (Figure 2), but development was not affected by the type of Cas9 mRNA or the concentration of Cas 9 mRNA that was injected (Figure 2). The goal was to determine if large deletions existed in the PCR amplicons by agarose gel electrophoresis. There were no differences in the percentage 


\section{THE NEXT GENERATION GENO/GRINDER ${ }^{\circledR}$ IS HERE!}

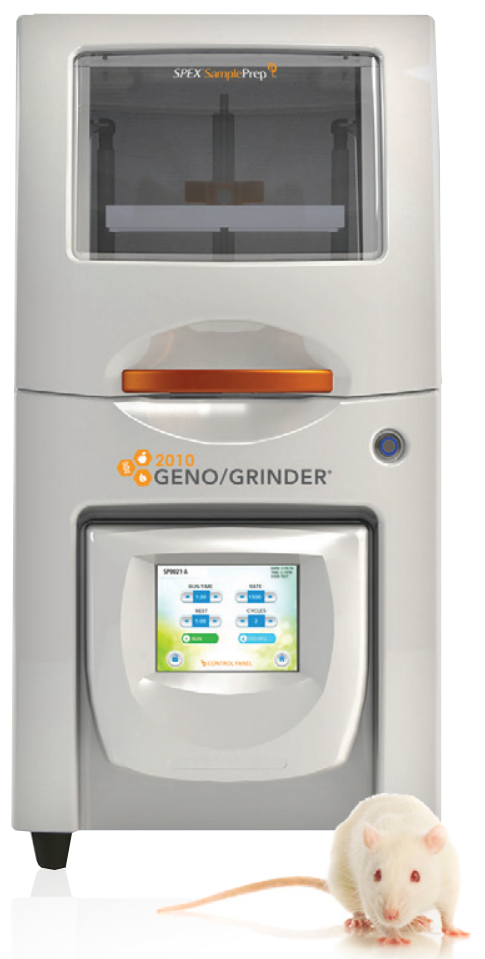

The Geno/Grinder ${ }^{\circledR}$ is a highthroughput plant \& animal tissue homogenizer with an adjustable clamp that accommodates up to six deep-well titer plates, or multiple sample tubes from $2 \mathrm{~mL}$ to $50 \mathrm{~mL}$. It is specifically designed for rapid cell disruption, lysis and tissue homogenization.

The new design incorporates a new front loading clamp and a programmable touch screen control panel that saves up to 500 protocols.

\section{SPEX SamplePrep P. (+1) 732-623-0465} learnmore@spex.com

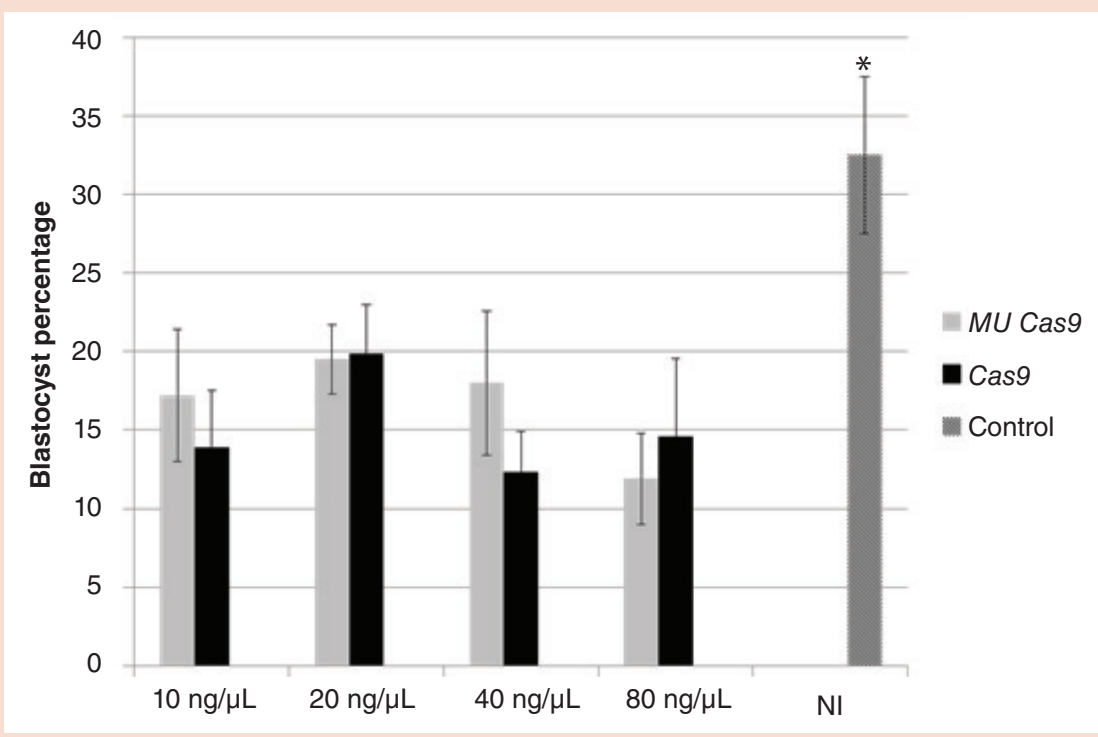

Figure 2. The effect of Cas9 type and concentration on blastocyst percentage. This data is derived from 3 replicates containing 196, 178; 198, 175; 204, 180; and 194, 175, injected zygotes respectively. NI refers to the non-injected control.

${ }^{*} p>0.05$.

\begin{tabular}{|c|c|c|c|c|}
\hline & \multicolumn{2}{|c|}{ Proprietary Gene X } & \multicolumn{2}{|c|}{$P A H$} \\
\hline Cas9 Source & $\begin{array}{l}\% \text { of Blastocysts } \\
\text { Modified } \pm \text { SE }\end{array}$ & $\begin{array}{l}\text { Number of Blasto- } \\
\text { cysts Assayed }\end{array}$ & $\begin{array}{l}\% \text { of Blastocysts } \\
\text { Modified } \pm \text { SE }\end{array}$ & $\begin{array}{l}\text { Number of Blasto- } \\
\text { cysts Assayed }\end{array}$ \\
\hline 20 ng/ $\mu$ L Cas 9 & $49 \% \pm 8 \%$ & $\mathrm{~N}=29$ & $47 \% \pm 5 \%$ & $\mathrm{~N}=37$ \\
\hline $20 \mathrm{ng} / \mu \mathrm{L}$ MU Cas 9 & $49 \% \pm 9 \%$ & $\mathrm{~N}=21$ & $38 \% \pm 14 \%$ & $N=35$ \\
\hline
\end{tabular}

Table 3. Analysis of gene modifications in blastocysts from zygote injections of commercially available Cas9 or $M U$ Cas 9 plus gRNAs.

of modifications as seen by gel imaging between the sources of Cas9 mRNA; however there was an increase in the percentage of modifications in the 80 $\mathrm{ng} / \mathrm{\mu l}$ concentration of $\mathrm{MU}$ Cas 9 mRNA compared to commercial Cas9 mRNA (Table 1). By assessing if large deletions existed in the PCR amplicons as a way to measure modification efficiency, we underestimated the number of true modifications present in each embryo and did not detect mosaicism.

To confirm our results, we assessed modifications by using gRNAs for two different genes (proprietary Gene $X$ and phenylalanine hydroxylase [PAH] Table 2). MU Cas 9 mRNA and Cas9 mRNA were injected $(20 \mathrm{ng} / \mu \mathrm{l})$ with different pairs of gRNAs and the resulting blastocysts were analyzed for modifications. There were no differences in the percentage of blastocysts that developed after injection by either MU Cas9 or Cas9 mRNA with the respective guides. Gene X had 16\% vs. $14 \%$ blastocyst development and $\mathrm{PAH}$ had $10 \%$ vs 9\% blastocyst development for Cas9 and MU Cas9, respec- tively. There was also no difference in the percent of blastocysts modified by injection of either Cas 9 mRNA with guides (Table 3).

We also wanted to determine if a $5^{\prime}$ cap was needed for this mRNA to be functional. We injected the same four guide RNAs from the first experiment and $20 \mathrm{ng} / \mu \mathrm{l}$ MU Cas9 mRNA_NC (without a $5^{\prime}$ cap) into zygotes. On day 7, blastocysts were collected and the gel shift assay showed that 18 of the 19 blastocysts collected were modified (94.7\%). This illustrates the functionality of an un-capped Cas 9 mRNA for use in zygote injections.

In summary, making Cas9 mRNA with a triple helical tail was efficient and results in genetic modification not different from commercial Cas 9 mRNA. When injected into zygotes, there is no difference in blastocyst development compared to the commercial Cas9 mRNA at the same concentration. There is also no difference in the percent of modifications between the two sources of Cas 9 mRNA, with the exception of the $80 \mathrm{ng} / \mu \mathrm{l}$ concen- 
tration where MU Cas9 mRNA had a higher rate. The successful generation of an alternative Cas 9 mRNA provides an attractive one-step mRNA protocol for use in zygote injections.

\section{Author contributions}

$\mathrm{BKR}, \mathrm{BPB}, \mathrm{RSP}$, and KDW designed the experiments. BKR performed the experiments. LDS, JAB., and SLM. assisted with IVF and injections. CWO and AMS assisted in developing gene assays. BKR and RSP wrote the manuscript with contributions from all authors.

\section{Acknowledgements}

This work was supported by the National Swine Research and Resource Center (NSRRC) U42 OD011140. This paper is subject to the NIH Public Access Policy.

\section{Competing interests}

The authors declare no competing interests.

\section{References}

Whitworth, K.M., et al., 2014. Use of the CRISPR/Cas9 system to produce genetically engineered pigs from in vitro-derived oocytes and embryos. Biol. Reprod. 91(3):78.

2. Whitworth, K.M., et al., 2017. Zygote injection of CRISPR/Cas9 RNA successfully modifies the target gene without delaying blastocyst development or altering the sex ratio in pigs. Transgenic Res. 2017. 26(1):97-107.

3. Sheets, T.P., et al., 2016. Somatic Cell Nuclear Transfer Followed by CRIPSR/Cas9 Microinjection Results in Highly Efficient Genome Editing in Cloned Pigs. Int. J. Mol. Sci. 17(12).

4. Wang, X., et al., 2015. Generation of genemodified goats targeting MSTN and FGF5 via zygote injection of CRISPR/Cas9 system. Sci. Rep. 5:13878.

5. Wang, Y., et al., 2015. Efficient generation of gene-modified pigs via injection of zygote with Cas9/sgRNA. Sci. Rep. 5:8256.

6. Marzluff, W.F., 2015. Metazoan replicationdependent histone mRNAs: a distinct set of RNA polymerase II transcripts. Curr. Opin. Cell. Biol. 17(3):274-280

7ilusz, J.E., et al., 2012. A triple helix stabilizes the 3' ends of long noncoding RNAs that lack poly(A) tails. Genes Dev. 26(21):2392-2407.

8. Cong, L., et al., 2013. Multiplex genome engineering using CRISPR/Cas systems. Science 339(6121):819-823.
9. Ran, F.A., et al., 2015. In vivo genome editing using Staphylococcus aureus Cas9. Nature 2015. 520:186.

10. Spate, L.D., et al., 2015. PS48 can replace bovine serum albumin in pig embryo culture medium, and improve in vitro embryo development by phosphorylating AKT. Mol. Reprod. Dev. 82(4):315-320.

First draft submitted: 7 November 2017; Accepted for publication: 19 February 2018

Address correspondence to Kevin D Wells, Division of Animal Science, National Swine Resource and Research Center, University of Missouri, Columbia, MO, USA.

E-mail: wellskev@missouri.edu

To purchase reprints of this article, contact: s.cavana@future-science.com

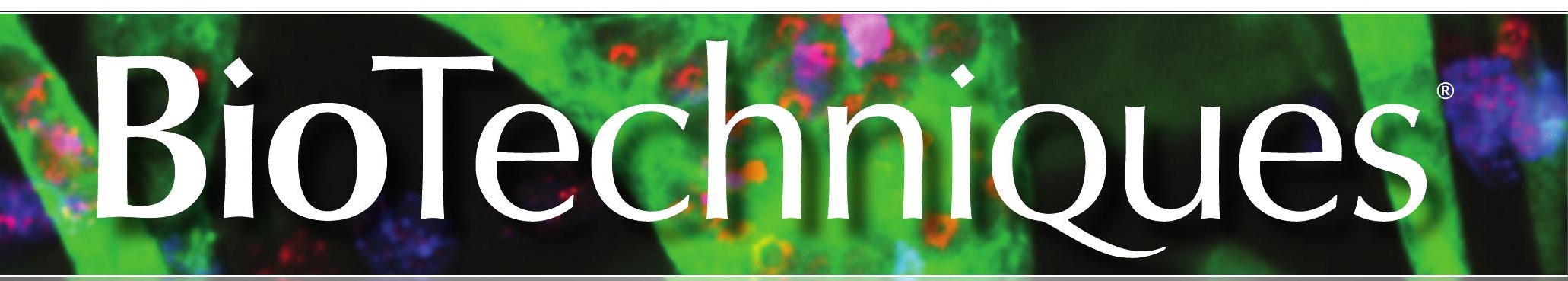

\section{The BioTechniques Vilobile App}

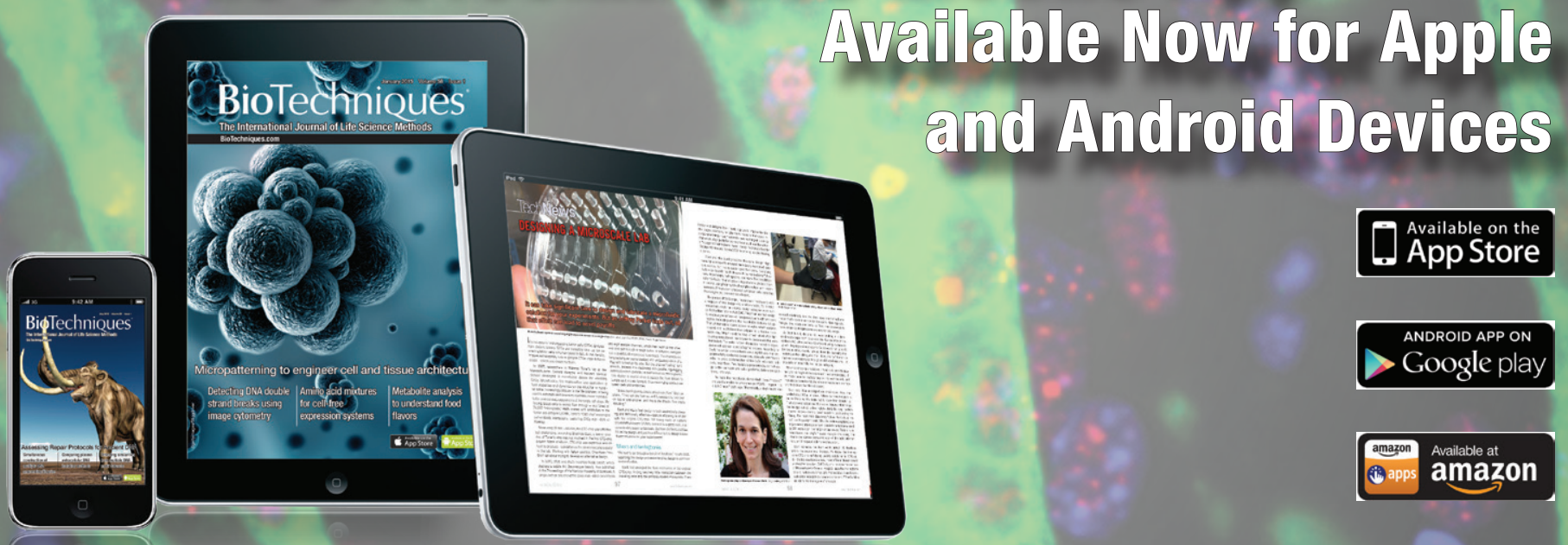

Visit BioTechniques.com/Digital to learn more and BioTechniques.com/Subscribe to receive monthly issue alerts. 